\title{
Characterizing Changes in Soybean Spectral Response Curves with Breeding Advancements
}

\author{
Brent S. Christenson, William T. Schapaugh, Jr., ${ }^{\star}$ \\ Nan An, Kevin P. Price, and Allan K. Fritz
}

\begin{abstract}
Soybean (Glycine max (L.) Merr.) crop yield has steadily increased in the past $60 \mathrm{yr}$ due in part to breeding advances. Relations between canopy spectral reflectance to specific plant functions may help characterize the impact of breeding on soybean cultivar development. The objectives of this study were to: 1) find specific regions of the soybean canopy spectral reflectance response curves that show genotypic differences; and 2) determine the effect of the breeding process on spectral reflectance response curves of soybean cultivars. Canopy spectral reflectance measurements were taken on 20 maturity group III (MGIII) and 20 maturity group IV (MGIV) soybean cultivars ranging in release year from 1923 to 2010 (arranged in a randomized complete block design) in 2011 and 2012 in Manhattan, KS. Large genotypic differences were found among cultivars, especially in the green $(500 \mathrm{~nm}-600 \mathrm{~nm})$, red $(600 \mathrm{~nm}-700$ $\mathrm{nm})$, and red-edge $(700 \mathrm{~nm}-730 \mathrm{~nm})$ portions of the spectra. Reflectance in the visible (VIS) $(400-700 \mathrm{~nm})$, red-edge $(700-730 \mathrm{~nm})$, and near-infrared (NIR) (730-1305) portions of the spectra varied with year of release (YOR) among cultivars. The more recently released cultivars tended to have lower reflectance values in the VIS and red-edge spectra portions and higher values in the NIR portion of the spectra than earlier-released cultivars. Results also indicate that spectral reflectance in the NIR portion of the spectra are highly confounded with maturity and other agronomic traits. These results indicate that breeding advancement has had an impact on canopy spectral reflectance curves and the VIS and red-edge portions of the spectra may be a source of variation for further cultivar development and advancement.
\end{abstract}

B.S. Christenson, W.T. Schapaugh, Jr. ${ }^{\star}$, N. An, K.P. Price, and A.K. Fritz, Dep. of Agronomy, Kansas State Univ., Manhattan, KS 66506. Received 29 Aug. 2013. `Corresponding author: (wts@ksu.edu).

Abbreviations: LAI, leaf area index; MG, maturity group; NDVI, normalized difference vegetation index; NIR, near-infrared; PAR, photosynthetically active radiation; VIS, visible; YOR, year of release.

$\mathrm{O}$ NE WAY to characterize plant phenotypes and function is by measuring canopy spectral reflectance. The amount of reflectance observed is a product of the leaf tissue/structure, cellular structure, and air-cell wall-protoplast-chloroplast interaction (Kumar and Silva, 1973). The visible (VIS) portion of the spectra $(400-700 \mathrm{~nm})$ has low reflectance due to the absorptive attributes of chlorophyll and other accessory pigments such as carotenoids, carotenes, and anthocyanins; however, the near-infrared (NIR) portion has a high reflectance caused by the scattering of light by cellular components and water. Many physiological parameters that show genotypic variation - most of which are pigment concentration and status-pertain to biochemical parameters (Reynolds et al., 2009). The photosynthetic capacity and efficiency of a plant is a function of the chlorophyll and other pigment content of the plant as well as membrane integrity. Chlorophyll status can also be used to estimate biomass production and photosynthetic capacity (Curran et al., 1990; Filella et al., 1995), and, because chlorophyll content is directly influenced by nitrogen, chlorophyll status estimation can relate directly to nitrogen status (Filella et al., 1995; Moran et al., 2000). By modeling the leaf structure and content, researchers can make inferences about

Published in Crop Sci. 54:1585-1597 (2014).

doi: 10.2135/cropsci2013.08.0575

Freely available online through the author-supported open-access option.

(C) Crop Science Society of America | 5585 Guilford Rd., Madison, WI 53711 USA

All rights reserved. No part of this periodical may be reproduced or transmitted in any form or by any means, electronic or mechanical, including photocopying, recording, or any information storage and retrieval system, without permission in writing from the publisher. Permission for printing and for reprinting the material contained herein has been obtained by the publisher. 
crop health and desired attributes, such as photosynthetic capabilities and water status (Thomas and Gausman, 1977; Wessman, 1990). Researchers also have estimated yield through other plant characteristics such as chlorosis (Adams et al., 1999), green cover (Dusek et al., 1985; Daughtry et al., 2000), chlorophyll (Datt, 1999; Daughtry et al., 2000), photosynthetically-active tissue (Wiegand et al., 1991), and water status (Prasad et al., 2007a, 2007b).

Chlorophylls have high absorbance in the red (600$700 \mathrm{~nm})$ and blue (400-500 nm) regions of the spectrum. Chlorophyll $b$ preferentially absorbs light in the 460 and $650 \mathrm{~nm}$ regions, chlorophyll $a$ absorbs in the 580, 630 , and $670 \mathrm{~nm}$ regions of the spectrum, and $415 \mathrm{~nm}$ is absorbed preferentially by both in soybean (Chappelle et al., 1992). Blue regions of the spectra also correlate to carotenoid-preferential absorption and therefore are not commonly used in chlorophyll concentration and status estimates (Sims and Gamon, 2002). The green and red regions of the spectrum-around $550 \mathrm{~nm}$ and $700 \mathrm{~nm}$, respectively-are primarily used, due to high chlorophyll concentrations needed to saturate these wavelengths (Sims and Gamon, 2002). Sims and Gamon (2002) correlated the $700 \mathrm{~nm}$ region to chlorophyll concentration, which is used by many researchers as a baseline for new chlorophyll indices. The ratio $\left(\mathrm{R}_{531}-\mathrm{R}_{570}\right) /\left(\mathrm{R}_{531}+\mathrm{R}_{570}\right)$ has been widely used to characterize leaf pigment status in many species (Gamon et al., 1992; Peñuelas et al., 1995). Chappelle et al. (1992) found that wavebands 650, 675, and $700 \mathrm{~nm}$ could be used to predict chlorophyll $\left(R^{2}=0.93\right)$ and $\beta$ carotene $\left(R^{2}=0.94\right)$ in soybean leaves. Gitelson and Merzlyak (1994) used $\left(\mathrm{R}_{780} / \mathrm{R}_{700}\right)$ and $\left(\mathrm{R}_{750}-\mathrm{R}_{705} /\right.$ $\mathrm{R}_{750}+\mathrm{R}_{705}$ ) to explain as much as $98 \%$ of the variability within chlorophyll content in chestnut and maple trees. Shanahan et al. (2001) explained from 70 to $92 \%$ of yield variability at mid-grain fill in corn using the green region and NIR. The normalized difference vegetation index (NDVI) derived by Deering (1978) and Tucker (1979) to estimate green biomass and intercepted PAR, defined as $\left(\mathrm{R}_{\mathrm{NIR}}-\mathrm{R}_{\mathrm{RED}} / \mathrm{R}_{\mathrm{NIR}}+\mathrm{R}_{\mathrm{RED}}\right)$, has been used to predict yield and other plant functions with many crops using hyperspectral and satellite imagery (Wiegand et al., 1991; Peñuelas et al., 1997; Ma et al., 2001; Shanahan et al., 2001; Royo et al., 2002; Royo et al., 2003; Prasad et al., 2007a, 2007b; Marti et al., 2007). Ma et al. (2001) explained up to $80 \%$ of the yield variability in soybean using 613 and $813 \mathrm{~nm}$. Others have also used the 550 and $700 \mathrm{~nm}$ regions of the spectrum when leaves are yellow-green (Gitelson and Merzlyak, 1996; Datt 1998; Gamon and Surfus, 1999; Sims and Gamon, 2002) to estimate chlorophyll status and yield, but high absorption in these regions may lead to saturation at relatively low chlorophyll concentrations. Gamon and Surfus (1999) developed a model and indices for anthocyanin concentration estimation based on a red-to-green ratio, $\mathrm{R}_{600}-\mathrm{R}_{700} / \mathrm{R}_{500}-\mathrm{R}_{600}$, and Viña and
Gitelson (2011) used broadband wavelengths to estimate anthocyanin status $\left(R^{2}=0.25-0.89\right)$ with peak absorption around 540 to $560 \mathrm{~nm}$.

Plant water status and stress also can be characterized through the preferential absorption of water and, more specifically, the hydroxyl ions within the electromagnetic spectrum (Peñuelas et al., 1993; Peñuelas et al., 1997; Gao, 1996; Serrano et al., 2000). The NIR (700-1100) and middle infrared (1100-2500 $\mathrm{nm}$ ) ranges have been shown to correlate well with water status or content of plants and wavelengths 970, 1240, 1400, and $2700 \mathrm{~nm}$ have been proposed as water absorption bands (Peñuelas et al., 1993; Gao, 1996; Gutierrez et al., 2010). Babar et al. (2006a, 2006b) used the wavelengths developed by Peñuelas et al. (1993) and found them useful for screening winter wheat (Triticum aestivum L.) genotypes for water stress and wateruse efficiency. Prasad et al. (2007a, 2007b) used the wavelengths 970, 920, 900, 880, and $850 \mathrm{~nm}$ to screen winter wheat lines under dryland conditions and found significant relationships with yield. Gao (1996) normalized wavebands $\left(\mathrm{R}_{860}-\mathrm{R}_{1240} / \mathrm{R}_{860}+\mathrm{R}_{1240}\right)$ and evaluated water stress in corn (Zea mays L.) and soybean, with favorable results.

Spectral reflectance correlated to specific plant functions may help characterize the impact of breeding on soybean cultivar development. The objectives of this study were: 1) to find specific regions of the soybean canopy spectral reflectance response curves that show genotypic differences and could be exploited for genotype distinction; and 2) to determine the effect of the breeding process on spectral response curves of soybean cultivars released from 1923 to 2010.

\section{MATERIALS AND METHODS Experimental and Field Design}

This study was conducted on soybean [Glycine max (L) Merr.] at the Kansas State University research farm south of Manhattan, KS, in 2011 and 2012. In 2011, experiments (field A-39 ${ }^{\circ}{ }^{\prime} 25^{\prime \prime}$ N 96 $37^{\prime} 46^{\prime \prime}$ W; elevation $322 \mathrm{~m}$ ) were conducted on a coarsesilty, mixed, superactive, nonacid, mesic Typic Udifluvents and coarse-silty, mixed, superactive, mesic Fluventic Hapludolls soil. In 2012, field B (39 $7^{\prime} 57^{\prime \prime}$ N 96 $97^{\circ} 5^{\prime \prime}$ W; elevation 322 m) was a fine-silty, mixed, superactive, mesic Fluventic Hapludolls and clayey over loamy, smectitic, mesic Fluvaquentic Hapludolls soil, and field C (39 $8^{\prime} 35^{\prime \prime}$ N 96 $37^{\prime} 46^{\prime \prime}$ W; elevation $322 \mathrm{~m}$ ) was a coarse-silty, mixed, superactive, nonacid, mesic Typic Udifluvents and coarse-silty, mixed, superactive, mesic Fluventic Hapludolls soil.

Twenty maturity group III (MGIII) and 20 maturity group IV (MGIV) soybean cultivars, ranging in release year from 1923 to 2010 (Table 1), were selected for release-year diversity out of the Soybean Genetic Gain Study coordinated by Dr. Brian Diers, University of Illinois. Selected cultivars were a mixture of private and public cultivars.

Replicated plots were planted on 23 May 2011 on field A, and 16 May 2012 and 4 June 2012 on fields B and C, respectively. Each experimental unit consisted of 4 rows $(3.4 \mathrm{~m}$ 
Table 1. Cultivars used for spectral data evaluation with year of release and 2-yr yield averages among maturity group III (MGIII) and maturity group IV (MGIV) cultivars.

\begin{tabular}{|c|c|c|c|c|c|}
\hline Cultivar & $\begin{array}{c}\text { Year of } \\
\text { Release }\end{array}$ & Yield & Cultivar & $\begin{array}{l}\text { Year of } \\
\text { Release }\end{array}$ & Yield \\
\hline & & tha $^{-1}$ & & & $\mathrm{t} \mathrm{ha}^{-1}$ \\
\hline AK (Harrow) & 1928 & 2.67 & Boone & 1935 & 2.34 \\
\hline Calland & 1968 & 2.95 & Chief & 1940 & 1.97 \\
\hline Dunfield & 1923 & 2.32 & Clark & 1953 & 2.70 \\
\hline IA 3010 & 1998 & 4.30 & Cutler & 1968 & 2.74 \\
\hline Illini & 1927 & 2.72 & Douglas & 1980 & 2.76 \\
\hline Lincoln & 1943 & 2.51 & Flyer & 1988 & 3.48 \\
\hline MACON & 1995 & 3.86 & KS4694 & 1993 & 3.24 \\
\hline NE3001 & 2004 & 3.59 & LD00-3309 & 2005 & 4.09 \\
\hline Private $3-1$ & 1978 & 3.80 & Macoupin & 1930 & 2.29 \\
\hline Private 3- 8 & 2002 & 4.08 & Private 4- 1 & 1985 & 3.54 \\
\hline Private 3- 9 & 1989 & 4.03 & Private 4- 4 & 2001 & 3.86 \\
\hline Private 3-12 & 1997 & 4.11 & Private 4- 6 & 1980 & 3.56 \\
\hline Private 3-13 & 2004 & 4.44 & Private 4-11 & 2000 & 4.04 \\
\hline Private 3-14 & 2007 & 4.63 & Private 4-12 & 1973 & 2.92 \\
\hline Private 3-15 & 1983 & 2.99 & Private 4-13 & 1984 & 3.60 \\
\hline Private 3-21 & 2001 & 4.30 & Private 4-19 & 2006 & 3.68 \\
\hline Private 3-23 & 2006 & 4.49 & Private $4-20$ & 2008 & 4.21 \\
\hline Shelby & 1958 & 2.81 & Private 4-21 & 2010 & 4.26 \\
\hline Wayne & 1964 & 2.92 & Private 4-22 & 2000 & 3.68 \\
\hline Williams & 1971 & 3.44 & Sparks & 1981 & 2.98 \\
\hline
\end{tabular}

long; spaced $76 \mathrm{~cm}$ apart). Cultivars were planted in separate well-watered and water-stressed environments arranged in 4 randomized complete blocks. In both years, weed pressure and fertility were not limiting. Flood irrigation was applied to the well-watered environments starting at reproductive stage R1 and continued weekly until R6 (Fehr and Caviness, 1977). In 2011, no supplemental irrigation was applied to the water-stressed environments. Due to extremely dry conditions, irrigation was applied once, shortly after R1 in 2012 to ensure crop development in the water-stressed environments.

\section{Phenotypic Traits}

Maturity, height, lodging, and seed yield data were taken on all plots during both seasons. Maturity was recorded when $95 \%$ of the pods had reached mature plant color. Height $(\mathrm{cm})$ was measured as the distance from the base of the plant to the top of the main stem. Lodging was scored on a scale of 1 to 5 , based on the amount of leaning or broken plants. Upright plants with no lean were scored as 1 ; other scores were $2=20^{\circ}$ lean, $3=45^{\circ}$ lean, $4=$ $60^{\circ}$ lean, and $5=$ flat on the ground. The center two rows of each plot were mechanically harvested using a 2-row plot combine. Seed yield was recorded as $\mathrm{t} \mathrm{ha}^{-1}$, adjusted to uniform moisture.

\section{Canopy Reflectance Measurements}

Canopy reflectance measurements were conducted using an ASD FieldSpec 3 spectroradiometer (Analytical Spectral Devices, Boulder, CO). Solar radiation reflecting back from the plant canopy was captured from 350 to $2500 \mathrm{~nm}$ in the electromagnetic spectrum using fiber optics with a $25^{\circ}$ field of view and sampling intervals of $1.4 \mathrm{~nm}$ between 350 and 1050 $\mathrm{nm}$ and $2 \mathrm{~nm}$ between 1050 and $2500 \mathrm{~nm}$. Moving averages were calculated automatically to achieve 1-nm-width continuous bands. A spectrolan (Spectrolan Labs) reflectance panel was used to calibrate the spectroradiometer with a dark current and white reference every 40 plots or when needed (ranging from every plot to 40 plot intervals, depending on field conditions) as well as used to create reflected light percentages. The sensor was mounted on an adjustable monopod pole and held vertically, approximately $1 \mathrm{~m}$ above the canopy to achieve approximately a $50-\mathrm{cm}$ diameter collection area. Two measurements were taken per plot on rows 2 and 3, excluding the first meter of each plot to eliminate border effect. Each measurement was the average of 10 scans, which was calculated automatically. Spectral data were collected on nearly cloud-free days within $\pm 2 \mathrm{~h}$ of solar noon. Measurements were collected weekly from R3 to R6 in 2011 and R2 to R6 in 2012, totaling three and four collection dates for the water-stressed and well-watered environments in 2011, respectively. In 2012, there were five collection dates for the well-watered MGIV environment on field B and four collection dates for the well-watered MGIII environment on field B and well-watered MGIII and MGIV environment on field C. There were three collection dates for the water-stressed environment on field B, but only two collection dates for the water-stressed environment on field $\mathrm{C}$ for each maturity group.

\section{Data Pretreatment}

Canopy reflectance data were initially trimmed from 350-2500 $\mathrm{nm}$ to $400-1310 \mathrm{~nm}$, which was necessary to eliminate noise from atmospheric absorption regions focused around the water absorption wavebands in the infrared and atmospheric scatter of blue color in the ultraviolet portion of the spectrum. Before averages were calculated for observation day and season totals, initial outlier control was implemented on each observation day's raw data. After outliers were identified and excluded from analysis, data were combined to form 10-nm-wide waveband region means to reduce the dataset size and eliminate some of the collinearity associated with hyperspectral reflectance data wavebands in close proximity (De Jong et al. 2003; Curran et al., 2001). Combined bands contain less variation from sample to sample than single-band measurements (Lin et al., 2012). Also, due to the high correlation with wavebands in close proximity, minimal information was thought to be lost due to waveband combination.

\section{Statistical Analyses}

All data were analyzed using SAS 9.2 (SAS Institute, 2008). The GLIMMIX procedure of SAS (SAS Institute, 2008) was used for analysis of variance (ANOVA) of yield, maturity, lodging, and height. Cultivar and environment were treated as fixed effects and replication nested within the environment was treated as a random effect. PROC GLM was used for ANOVA of the waveband regions. Spectral data were analyzed for differences among cultivars using the genotype $\times$ environment interaction as the error term. PROC CORR was used to characterize the relationship between (YOR), maturity, lodging, and height with each waveband region. PROC REG was used to characterize the significance of the YOR, waveband region relationship when maturity, lodging, and height were accounted for in a regression model. 


\section{RESULTS AND DISCUSSION \\ Cultivar Performance for Yield and Reflectance Data}

Cultivars differed $(P \leq 0.0001)$ in average seed yield across the six environments in both maturity groups (Table 1). Seed yield ranged from 2.32 to $4.63 \mathrm{t} \mathrm{ha}^{-1}$ and 1.97 to $4.26 \mathrm{t} \mathrm{ha}^{-1}$ for the MGIII and MGIV cultivars, respectively. Seed yield increased with year of release by 0.0238 and $0.0255 \mathrm{t} \mathrm{ha}^{-1} \mathrm{yr}^{-1}$ and had coefficient of determination values of 82 and $86 \%$ between yield and YOR in MGIII and MGIV, respectively (Fig. 1). Yield increases are consistent with observations of short-season soybean covering 58 yr of cultivar development (Morrison et al., 1999). Differences were noted among cultivars and environments for seed yield, and a significant genotype $\times$ environment interaction was observed $(P<0.0001)$ in both maturity groups (Table 2). The genotype $\times$ environment $(G \times E)$ interaction variances were quite low compared with the total phenotypic variation of each maturity group because the cultivars tested were genetically diverse with a large gradient between high-yielding and low-yielding cultivars.

Cultivar differences $(P<0.05)$ were observed for individual waveband regions in both maturity groups, with the exception of $905 \mathrm{~nm}$ in MGIII (Table 3). The VIS portion of the spectrum (405-695 nm) had greater genotypic differences than the NIR (705-1305 nm) in both maturity groups. The greatest genotypic differences (based on $\mathrm{F}$ values) were observed in the green and red portions of the spectrum in both maturity groups. In the MGIII and MGIV experiments, the 735 to $1135 \mathrm{~nm}$ region had the smallest differences among cultivars of all portions of the spectrum. Large $G \times E$ interactions and high variability in the NIR from cellular scatter and soil confounds may account for the decreased genotypic differences observed for this region. The MGIV cultivars had higher genotypic differences in reflectance values than the MGIII cultivars because $G \times E$ interactions constituted less of the total phenotypic variation among MGIV cultivars than MGIII cultivars.

Spectral responses varied based on YOR in both maturity groups. The earliest released cultivars tended to have higher reflectance values in the VIS portion of the spectrum and lower values in the NIR than the most recently released cultivars (Fig. 2A, 2B, 3A and 3B). High values for the blue and red portions suggests that chlorophyll and other associated pigments are not as plentiful or efficient in the earlier released cultivars, and higher reflectance values in the green correlates with more yellow pigment and potentially more foliar diseases or issues contributing to decreased plant function. These results are consistent with trends observed in soybean (Glycine $\max (\mathrm{L})$. Merr.) (Ma et al., 2001), corn (Zea mays L.) (Chang et al., 2003; Weber et al., 2012), wheat (Triticum aesitivum L.) (Hansen et al., 2002), and rice (Oryza sativa L.) (Lin et al., 2012). Higher

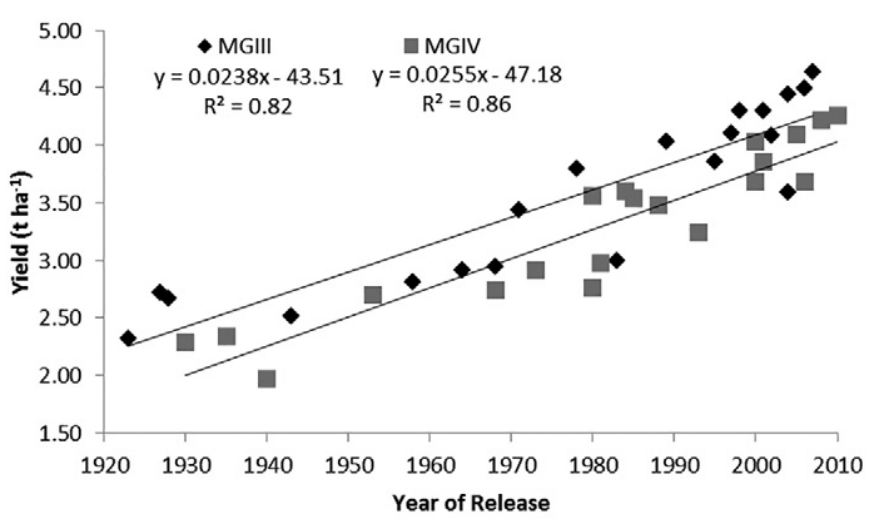

Figure 1. Relationship between year of release and two-year yield means for maturity group III (MGIII) and maturity group IV (MGIV) experiments. Least square lines with equations and coefficients of determination $\left(\mathrm{R}^{2}\right)$.

Table 2. Analysis of variance for seed yield of maturity group III (MGIII) and maturity group IV (MGIV) cultivars.

\begin{tabular}{lrrr}
\hline \multicolumn{1}{c}{ Source } & D.F. & F Value & Pr $>$ F \\
\hline MGIII & & & \\
$\quad$ Genotype (G) & 19 & 111.57 & $<0.0001$ \\
Environment (E) & 5 & 109.10 & $<0.0001$ \\
G $\times$ E & 95 & 3.11 & $<0.0001$ \\
MGIV & & & \\
Genotype (G) & 19 & 95.98 & $<0.0001$ \\
Environment (E) & 5 & 82.76 & $<0.0001$ \\
G $\times$ E & 95 & 3.90 & $<0.0001$ \\
\hline
\end{tabular}

† Degrees of freedom

reflectance in the NIR suggests more biomass production or denser canopy and more intact cellular structure in the more recently released cultivars (Sims and Gamon, 2002; Royo et al., 2003). Among the MGIV cultivars, greater separation was observed between cultivars in the NIRan area correlated with brown pigmentation, biomass production, cellular structure, and water status - than among the MGIII cultivars (Fig. 2B and 3B). These results could be due to MGIV cultivars being later in maturity than MGIII cultivars, resulting in greater biomass accumulation and less senescence during later observation days.

\section{Spectral Reflectance In Relation to Year of Release and Agronomic Traits}

Many of the cultivar differences in canopy spectral reflectance were highly correlated $(r)$ to YOR and maturity, lodging, and height (Fig. 4 and 5). The highest $r$ values were observed in the VIS portion among both MGs for YOR and lodging (Fig. 4 and 5). Reflectance in the blue (405-495), green (505-595 nm), red (605-695 nm), and red-edge $(705-735 \mathrm{~nm})$ regions of the spectrum $(r=-0.53$ to $-0.77, r=-0.79$ to $-0.83, r=-0.76$ to -0.85 , and $r=$ -0.76 to -0.88 , respectively) decreased as YOR increased. Canopy reflectance tended to increase as lodging increased in the VIS spectra ( $r=0.34$ to 0.66$)$. The VIS portions 
Table 3. Waveband regions $(\mathrm{nm})$ with genotypic differences from analysis of variance $(N=20)$ and results from stepwise regression analysis with year of release, maturity, height, and lodging as predictor variables in an optimized stepwise model as well as maturity, lodging, and height forced into the model before year of release for maturity group III (MGIII) and maturity group IV (MGIV) cultivars.

\begin{tabular}{|c|c|c|c|c|c|c|c|c|c|c|}
\hline \multirow[b]{3}{*}{$\begin{array}{l}\text { Wave- } \\
\text { band }\end{array}$} & \multicolumn{5}{|c|}{ MGIII } & \multicolumn{5}{|c|}{ MGIV } \\
\hline & \multirow[b]{2}{*}{ F-Value } & \multicolumn{2}{|c|}{$\begin{array}{c}\text { Optimized } \\
\text { Stepwise Model }\end{array}$} & \multicolumn{2}{|c|}{$\begin{array}{c}\text { Maturity, Lodging, } \\
\text { Height Included First } \\
\end{array}$} & \multirow[b]{2}{*}{ F-Value } & \multicolumn{2}{|c|}{$\begin{array}{c}\text { Optimized } \\
\text { Stepwise Model }\end{array}$} & \multicolumn{2}{|c|}{$\begin{array}{l}\text { Maturity, Lodging, } \\
\text { Height Included First }\end{array}$} \\
\hline & & $\begin{array}{c}\text { YOR }^{\dagger} \\
\text { Partial } R^{2}\end{array}$ & Model $R^{2}$ & $\begin{array}{c}\text { YOR } \\
\text { Partial } R^{2}\end{array}$ & Model $R^{2}$ & & $\begin{array}{c}\text { YOR } \\
\text { Partial } R^{2}\end{array}$ & Model $R^{2}$ & $\begin{array}{c}\text { YOR } \\
\text { Partial } R^{2}\end{array}$ & Model $R^{2}$ \\
\hline 405 & $20.61^{\star \star}$ & $0.37^{\star \star}$ & $0.47^{\star \star}$ & $0.12^{\ddagger}$ & $0.48^{\star}$ & $13.47^{\star \star}$ & NS§ & $0.40^{\star \star}$ & $0.16^{\star}$ & $0.57^{\star \star}$ \\
\hline 415 & $19.96^{\star \star}$ & $0.39^{\star \star}$ & $0.39^{\star \star}$ & $0.11^{\ddagger}$ & $0.48^{\star}$ & $13.63^{\star \star}$ & NS & $0.41^{\star \star}$ & $0.15^{\star}$ & $0.58^{\star \star}$ \\
\hline 425 & $17.41^{\star \star}$ & $0.45^{\star *}$ & $0.45^{\star \star}$ & $0.11^{\ddagger}$ & $0.52^{\star}$ & $12.54^{\star \star}$ & NS & $0.40^{\star *}$ & $0.15^{\star}$ & $0.58^{\star *}$ \\
\hline 435 & $15.80^{* *}$ & $0.51^{* *}$ & $0.51^{\star \star}$ & $0.11^{\ddagger}$ & $0.56^{* *}$ & $11.41^{\star *}$ & NS & $0.39^{* *}$ & $0.16^{\star}$ & $0.57^{* *}$ \\
\hline 445 & $15.26^{\star \star}$ & $0.54^{\star \star}$ & $0.54^{\star \star}$ & $0.10^{\ddagger}$ & $0.58^{* \star}$ & $10.85^{\star \star}$ & NS & $0.37^{\star \star}$ & $0.16^{*}$ & $0.56^{\star}$ \\
\hline 455 & $15.11^{\star \star}$ & $0.55^{\star \star}$ & $0.55^{\star \star}$ & $0.10^{\ddagger}$ & $0.59^{\star *}$ & $10.68^{\star \star}$ & NS & $0.37^{* \star}$ & $0.15^{\star}$ & $0.55^{\star}$ \\
\hline 465 & $15.41^{\star *}$ & $0.56^{\star *}$ & $0.56^{\star *}$ & $0.10^{\ddagger}$ & $0.60^{* *}$ & $10.59^{\star \star}$ & NS & $0.37^{* *}$ & $0.15^{\star}$ & $0.54^{\star}$ \\
\hline 475 & $15.78^{\star \star}$ & $0.57^{* *}$ & $0.57^{\star \star}$ & $0.09^{\ddagger}$ & $0.61^{\star \star}$ & $10.58^{\star \star \star}$ & NS & $0.37^{\star \star}$ & $0.15^{\star}$ & $0.54^{\star}$ \\
\hline 485 & $16.09^{\star *}$ & $0.58^{\star *}$ & $0.58^{* \star}$ & $0.09 \ddagger$ & $0.62^{* *}$ & $10.56^{\star \star}$ & NS & $0.37^{\star *}$ & $0.14^{*}$ & $0.54^{\star}$ \\
\hline 495 & $16.90^{* *}$ & $0.59^{* \star}$ & $0.59^{\star *}$ & $0.09 \ddagger$ & $0.63^{\star *}$ & $10.88^{* \star *}$ & NS & $0.39^{\star *}$ & $0.14^{\star}$ & $0.55^{\star}$ \\
\hline 505 & $18.66^{\star \star}$ & $0.62^{\star \star}$ & $0.62^{\star \star}$ & $0.08^{\ddagger}$ & $0.66^{\star \star}$ & $13.60^{\star \star}$ & NS & $0.44^{\star \star}$ & $0.14^{*}$ & $0.58^{\star \star}$ \\
\hline 515 & $22.73^{\star \star}$ & $0.66^{* *}$ & $0.66^{\star *}$ & $0.08^{\ddagger}$ & $0.70^{* *}$ & $15.00^{* *}$ & $0.53^{* *}$ & $0.62^{* *}$ & $0.16^{\star}$ & $0.66^{\star *}$ \\
\hline 525 & $29.10^{\star *}$ & $0.66^{\star *}$ & $0.66^{\star \star}$ & $0.07^{\ddagger}$ & $0.71^{* *}$ & $17.86^{\star \star}$ & $0.63^{\star \star}$ & $0.71^{\star *}$ & $0.17^{\star *}$ & $0.73^{\star *}$ \\
\hline 535 & $32.83^{\star *}$ & $0.66^{\star \star}$ & $0.66^{\star *}$ & $0.07^{\ddagger}$ & $0.71^{\star *}$ & $18.87^{\star \star}$ & $0.67^{\star \star}$ & $0.74^{\star *}$ & $0.18^{* *}$ & $0.75^{\star \star}$ \\
\hline 545 & $34.42^{\star *}$ & $0.66^{\star *}$ & $0.70^{* *}$ & $0.06^{\ddagger}$ & $0.71^{\star *}$ & $19.32^{\star \star}$ & $0.69^{* *}$ & $0.75^{\star \star}$ & $0.18^{* *}$ & $0.76^{\star \star}$ \\
\hline 555 & $34.77^{* *}$ & $0.66^{* *}$ & $0.70^{\star \star}$ & $0.06^{\ddagger}$ & $0.72^{* *}$ & $19.46^{\star \star}$ & $0.69^{* *}$ & $0.76^{\star *}$ & $0.18^{\text {** }}$ & $0.76^{\star *}$ \\
\hline 565 & $33.83^{\star *}$ & $0.67^{* *}$ & $0.71^{\star *}$ & $0.06^{\ddagger}$ & $0.73^{* *}$ & $19.62^{\star \star}$ & $0.70^{\star \star}$ & $0.75^{\text {** }}$ & $0.17^{* *}$ & $0.76^{\star *}$ \\
\hline 575 & $31.08^{\star \star}$ & $0.69^{* \star}$ & $0.73^{\star *}$ & $0.06^{\ddagger}$ & $0.74^{\star *}$ & $19.27^{\star \star}$ & $0.69^{* *}$ & $0.74^{\star *}$ & $0.16^{\star *}$ & $0.75^{\star \star}$ \\
\hline 585 & $28.34^{\star \star}$ & $0.69^{\star \star}$ & $0.74^{\star \star}$ & $0.07^{\ddagger}$ & $0.75^{\star \star}$ & $18.63^{\star *}$ & $0.68^{\star *}$ & $0.73^{\star *}$ & $0.15^{\star *}$ & $0.74^{\star *}$ \\
\hline 595 & $26.62^{\star \star}$ & $0.69^{\star \star}$ & $0.74^{\star \star}$ & $0.07^{\ddagger}$ & $0.76^{\star *}$ & $18.02^{\star \star}$ & $0.67^{\star \star}$ & $0.71^{\star \star}$ & $0.15^{\star *}$ & $0.73^{\text {** }}$ \\
\hline 605 & $25.23^{\star \star}$ & $0.70^{* *}$ & $0.75^{\star *}$ & $0.07^{\ddagger}$ & $0.76^{* *}$ & $17.53^{\star \star}$ & $0.66^{* *}$ & $0.70^{* *}$ & $0.15^{\star *}$ & $0.72^{* *}$ \\
\hline 615 & $22.78^{\star *}$ & $0.70^{* *}$ & $0.75^{\star \star}$ & $0.07^{\ddagger}$ & $0.76^{\star *}$ & $16.52^{\star \star}$ & $0.64^{\star *}$ & $0.68^{* *}$ & $0.14^{*}$ & $0.70^{\star *}$ \\
\hline 625 & $21.12^{\star *}$ & $0.70^{\star *}$ & $0.76^{\star \star}$ & $0.07^{\ddagger}$ & $0.76^{\star *}$ & $15.64^{\star *}$ & $0.63^{\star *}$ & $0.63^{\star *}$ & $0.13^{*}$ & $0.69^{\star *}$ \\
\hline 635 & $20.46^{\star \star}$ & $0.70^{* *}$ & $0.76^{\star \star}$ & $0.07^{\ddagger}$ & $0.76^{\star *}$ & $15.22^{* \star}$ & $0.62^{* *}$ & $0.62^{\star \star}$ & $0.13^{*}$ & $0.68^{\star *}$ \\
\hline 645 & $19.29^{\star *}$ & $0.68^{\star *}$ & $0.74^{\star \star}$ & $0.07^{\ddagger}$ & $0.75^{\star \star}$ & $13.82^{\star *}$ & $0.58^{* *}$ & $0.58^{* *}$ & $0.12^{*}$ & $0.65^{\star \star}$ \\
\hline 655 & $17.94^{\star \star}$ & $0.67^{\star *}$ & $0.73^{\star *}$ & $0.07^{\ddagger}$ & $0.74^{* *}$ & $12.44^{\star *}$ & $0.53^{\star *}$ & $0.53^{\star *}$ & $0.12^{*}$ & $0.62^{* *}$ \\
\hline 665 & $17.11^{\star \star}$ & $0.66^{\star *}$ & $0.72^{* *}$ & $0.07^{\ddagger}$ & $0.73^{* *}$ & $11.12^{\star \star}$ & $0.48^{* *}$ & $0.48^{\star *}$ & $0.12^{\ddagger}$ & $0.58^{* *}$ \\
\hline 675 & $17.33^{\star *}$ & $0.66^{\star *}$ & $0.71^{\star \star}$ & $0.08^{\ddagger}$ & $0.71^{\star *}$ & $10.61^{\star \star}$ & $0.45^{\star \star}$ & $0.45^{\star *}$ & $0.12^{\ddagger}$ & $0.56^{\star}$ \\
\hline 685 & $17.52^{\star *}$ & $0.67^{\star *}$ & $0.72^{* \star}$ & $0.08^{\ddagger}$ & $0.73^{* *}$ & $11.48^{\star \star}$ & $0.50^{\star \star}$ & $0.50^{\star *}$ & $0.13^{*}$ & $0.59^{\star *}$ \\
\hline 695 & $22.96^{\star \star}$ & $0.72^{\text {** }}$ & $0.77^{* *}$ & $0.07^{\ddagger}$ & $0.78^{* *}$ & $17.13^{\star \star}$ & $0.70^{\star *}$ & $0.70^{* *}$ & $0.15^{\text {** }}$ & $0.74^{\star \star}$ \\
\hline 705 & $37.14^{\star *}$ & $0.70^{* *}$ & $0.75^{\star \star}$ & $0.07^{\ddagger}$ & $0.77^{\star *}$ & $20.73^{\star \star}$ & $0.77^{\star \star}$ & $0.77^{\star \star}$ & $0.19^{\star *}$ & $0.80^{* *}$ \\
\hline 715 & $38.07^{\star \star}$ & $0.65^{\star \star}$ & $0.71^{\star \star}$ & $0.08^{\ddagger}$ & $0.72^{* *}$ & $18.55^{\star \star}$ & $0.75^{\star \star}$ & $0.78^{* *}$ & $0.21^{\text {** }}$ & $0.79^{* *}$ \\
\hline 725 & $22.66^{\star \star}$ & $0.58^{\star *}$ & $0.64^{* *}$ & $0.08^{\ddagger}$ & $0.65^{\star *}$ & $12.10^{\star *}$ & $0.62^{* *}$ & $0.68^{* *}$ & $0.23^{\star *}$ & $0.70^{* *}$ \\
\hline 735 & $8.69^{\star \star}$ & $0.42^{*}$ & $0.50^{* *}$ & $0.09 \ddagger$ & $0.52^{\star}$ & $5.41^{\star \star}$ & $0.27^{\star}$ & $0.44^{\star *}$ & $0.20^{\star}$ & $0.47^{\star}$ \\
\hline 745 & $3.12^{* \star}$ & NS & $0.16^{\ddagger}$ & NS & NS & $3.13^{\star \star}$ & NS & $0.22^{*}$ & NS & NS \\
\hline 755 & $1.96^{\star}$ & NS & NS & NS & NS & $3.19^{\star *}$ & NS & $0.42^{* *}$ & NS & $0.44^{*}$ \\
\hline 765 & $1.85^{\star}$ & NS & $0.22^{*}$ & NS & $0.33^{\ddagger}$ & $3.41^{\star *}$ & NS & $0.48^{\star *}$ & NS & $0.53^{\star *}$ \\
\hline 775 & $1.96^{\star}$ & NS & $0.27^{\star}$ & NS & $0.39^{\star}$ & $3.61^{\star \star}$ & NS & $0.55^{\star *}$ & NS & $0.55^{\star \star}$ \\
\hline 785 & $1.99^{*}$ & NS & $0.29^{\star}$ & NS & $0.40^{*}$ & $3.68^{* *}$ & NS & $0.56^{\star *}$ & NS & $0.56^{\star *}$ \\
\hline 795 & $2.00^{*}$ & NS & $0.29^{*}$ & NS & $0.41^{*}$ & $3.70^{* \star}$ & NS & $0.56^{* *}$ & NS & $0.56^{\star *}$ \\
\hline 805 & $2.01^{\star}$ & NS & $0.29^{*}$ & NS & $0.41^{*}$ & $3.73^{\star *}$ & NS & $0.56^{* *}$ & NS & $0.56^{\star *}$ \\
\hline 815 & $1.97^{\star}$ & NS & $0.29^{\star}$ & NS & $0.40^{*}$ & $3.71^{\star *}$ & NS & $0.55^{\star *}$ & NS & $0.56^{\star *}$ \\
\hline 825 & $2.00^{*}$ & NS & $0.28^{*}$ & NS & $0.39^{\star}$ & $3.74^{\star \star}$ & NS & $0.55^{\star *}$ & NS & $0.55^{\star \star}$ \\
\hline 835 & $2.04^{\star \star}$ & NS & $0.28^{*}$ & NS & $0.39^{\star}$ & $3.80^{\star *}$ & NS & $0.54^{\star \star}$ & NS & $0.54^{\star *}$ \\
\hline 845 & $2.10^{\star *}$ & NS & $0.28^{*}$ & NS & $0.39^{\star}$ & $3.85^{\star *}$ & NS & $0.54^{* *}$ & NS & $0.54^{* *}$ \\
\hline 855 & $2.12^{\star \star}$ & NS & $0.27^{*}$ & NS & $0.39^{\star}$ & $3.89^{\star \star}$ & NS & $0.53^{\star \star}$ & NS & $0.53^{\star *}$ \\
\hline 865 & $2.15^{\star \star}$ & NS & $0.27^{*}$ & NS & $0.39^{\star}$ & $3.92^{* *}$ & NS & $0.53^{\star *}$ & NS & $0.53^{\star *}$ \\
\hline 875 & $2.17^{\star \star}$ & NS & $0.26^{\star}$ & NS & $0.38^{\star}$ & $3.95^{\star \star}$ & NS & $0.52^{\star *}$ & NS & $0.52^{\star \star}$ \\
\hline 885 & $2.16^{\star *}$ & NS & $0.26^{\star}$ & NS & $0.38^{\star}$ & $3.97^{\star \star}$ & NS & $0.52^{\star *}$ & NS & $0.52^{\star \star}$ \\
\hline
\end{tabular}




\begin{tabular}{|c|c|c|c|c|c|c|c|c|c|c|}
\hline \multirow[b]{3}{*}{$\begin{array}{l}\text { Wave- } \\
\text { band }\end{array}$} & \multicolumn{5}{|c|}{ MGIII } & \multicolumn{5}{|c|}{ MGIV } \\
\hline & \multirow[b]{2}{*}{ F-Value } & \multicolumn{2}{|c|}{$\begin{array}{c}\text { Optimized } \\
\text { Stepwise Model }\end{array}$} & \multicolumn{2}{|c|}{$\begin{array}{l}\text { Maturity, Lodging, } \\
\text { Height Included First }\end{array}$} & \multirow[b]{2}{*}{ F-Value } & \multicolumn{2}{|c|}{$\begin{array}{c}\text { Optimized } \\
\text { Stepwise Model }\end{array}$} & \multicolumn{2}{|c|}{$\begin{array}{l}\text { Maturity, Lodging, } \\
\text { Height Included First }\end{array}$} \\
\hline & & $\begin{array}{c}\text { YOR }^{\dagger} \\
\text { Partial } R^{2}\end{array}$ & Model $R^{2}$ & $\begin{array}{c}\text { YOR } \\
\text { Partial } R^{2}\end{array}$ & Model $R^{2}$ & & $\begin{array}{c}\text { YOR } \\
\text { Partial } R^{2}\end{array}$ & Model $R^{2}$ & $\begin{array}{c}\text { YOR } \\
\text { Partial } R^{2}\end{array}$ & Model $R^{2}$ \\
\hline 895 & $1.97^{*}$ & NS & $0.27^{\star}$ & NS & $0.38^{*}$ & $3.95^{\star \star}$ & NS & $0.51^{\star \star}$ & NS & $0.51^{\star \star}$ \\
\hline 905 & 1.59 & NS & $0.28^{\star}$ & NS & $0.49^{*}$ & $3.96^{\star *}$ & NS & $0.50^{* *}$ & NS & $0.50^{\star \star}$ \\
\hline 915 & $1.73^{*}$ & NS & $0.26^{\star}$ & NS & $0.38^{\star}$ & $3.96^{\star \star}$ & NS & $0.49^{\star \star}$ & NS & $0.50^{\star \star}$ \\
\hline 925 & $1.87^{\star}$ & NS & $0.23^{*}$ & NS & $0.35^{\ddagger}$ & $4.01^{\star \star}$ & NS & $0.48^{\star *}$ & NS & $0.48^{*}$ \\
\hline 935 & $2.05^{\star}$ & NS & $0.19^{\star}$ & NS & $0.28^{\ddagger}$ & $3.84^{\star \star}$ & NS & $0.40^{\star *}$ & NS & $0.47^{\star}$ \\
\hline 945 & $2.11^{\star \star}$ & NS & NS & NS & NS & $3.93^{\star \star}$ & NS & $0.38^{* *}$ & NS & $0.44^{*}$ \\
\hline 955 & $2.23^{\star \star}$ & NS & NS & NS & NS & $4.01^{\star \star}$ & NS & $0.34^{\star \star}$ & NS & $0.39^{*}$ \\
\hline 965 & $2.41^{\star \star}$ & NS & NS & NS & NS & $4.14^{\star \star}$ & NS & $0.31^{\star \star}$ & NS & $0.35^{\ddagger}$ \\
\hline 975 & $2.56^{\star \star}$ & NS & NS & NS & NS & $4.23^{\star \star}$ & NS & $0.30^{\star}$ & NS & $0.34^{\ddagger}$ \\
\hline 985 & $2.63^{\star *}$ & NS & NS & NS & NS & $4.30^{* *}$ & NS & $0.30^{\star}$ & NS & $0.34^{\ddagger}$ \\
\hline 995 & $2.67^{\star \star}$ & NS & NS & NS & NS & $4.34^{\star \star}$ & NS & $0.30^{*}$ & NS & $0.34^{\ddagger}$ \\
\hline 1005 & $2.67^{\star \star}$ & NS & NS & NS & NS & $4.66^{\star \star}$ & NS & $0.29^{*}$ & NS & $0.33^{\ddagger}$ \\
\hline 1015 & $2.66^{\star \star}$ & NS & NS & NS & NS & $4.68^{\star \star}$ & NS & $0.30^{*}$ & NS & $0.35^{\ddagger}$ \\
\hline 1025 & $2.65^{\star \star}$ & NS & NS & NS & NS & $4.69^{* \star}$ & NS & $0.31^{*}$ & NS & $0.36^{\ddagger}$ \\
\hline 1035 & $2.64^{\star \star}$ & NS & NS & NS & NS & $4.70^{\star \star}$ & NS & $0.32^{*}$ & NS & $0.37^{\ddagger}$ \\
\hline 1045 & $2.64^{\star \star}$ & NS & NS & NS & NS & $4.70^{\star \star}$ & NS & $0.33^{*}$ & NS & $0.39^{*}$ \\
\hline 1055 & $2.63^{\star \star}$ & NS & NS & NS & NS & $4.70^{\star \star}$ & NS & $0.33^{*}$ & NS & $0.39^{*}$ \\
\hline 1065 & $2.61^{\star \star}$ & NS & NS & NS & $0.28^{\ddagger}$ & $4.70^{\star \star}$ & NS & $0.33^{*}$ & NS & $0.40^{\star}$ \\
\hline 1075 & $2.58^{\star \star}$ & NS & $0.29^{*}$ & $0.08^{\ddagger}$ & $0.29^{\ddagger}$ & $4.70^{\star \star}$ & NS & $0.33^{*}$ & NS & $0.40^{*}$ \\
\hline 1085 & $2.34^{\star \star}$ & NS & $0.34^{*}$ & $0.15^{\ddagger}$ & $0.49^{\star}$ & $4.70^{\star \star}$ & NS & $0.33^{*}$ & NS & $0.39^{*}$ \\
\hline 1095 & $2.08^{\star \star}$ & NS & $0.28^{\star}$ & $0.25^{\star \star}$ & $0.62^{\star \star}$ & $4.69^{\star \star}$ & NS & $0.32^{*}$ & NS & $0.38^{*}$ \\
\hline 1105 & $2.39^{* \star}$ & NS & 0.31 & $0.17^{\star}$ & $0.48^{*}$ & $4.67^{\star \star}$ & NS & $0.31^{\star}$ & NS & $0.37^{\ddagger}$ \\
\hline 1115 & $2.57^{\star \star}$ & NS & 0.23 & $0.11^{\ddagger}$ & $0.34^{\ddagger}$ & $4.61^{\star \star}$ & NS & $0.30^{\star}$ & NS & $0.36^{\ddagger}$ \\
\hline 1125 & $2.60^{* *}$ & NS & NS & NS & NS & $4.51^{\star \star}$ & NS & $0.28^{\star}$ & NS & $0.33^{\ddagger}$ \\
\hline 1135 & $2.90^{\star \star}$ & NS & NS & NS & NS & $4.65^{\star \star}$ & NS & $0.23^{*}$ & NS & NS \\
\hline 1145 & $3.38^{\star *}$ & NS & NS & NS & NS & $4.81^{\star \star}$ & NS & $0.17^{\ddagger}$ & NS & NS \\
\hline 1155 & $4.26^{\star \star}$ & $0.15^{\ddagger}$ & $0.15^{\ddagger}$ & NS & NS & $5.08^{\star \star}$ & NS & $0.12^{\ddagger}$ & NS & NS \\
\hline 1165 & $4.56^{\star \star}$ & $0.17^{*}$ & $0.17^{\ddagger}$ & NS & NS & $5.24^{\star \star}$ & NS & NS & NS & NS \\
\hline 1175 & $4.74^{\star \star}$ & $0.18^{*}$ & $0.18^{\ddagger}$ & $0.17^{\ddagger}$ & $0.35^{\ddagger}$ & $5.32^{\star \star}$ & NS & NS & NS & NS \\
\hline 1185 & $4.86^{\star *}$ & $0.19^{*}$ & $0.19^{*}$ & $0.17^{\ddagger}$ & $0.35^{\ddagger}$ & $5.37^{\star \star}$ & NS & NS & NS & NS \\
\hline 1195 & $4.94^{\star \star}$ & $0.20^{*}$ & $0.20^{*}$ & $0.17^{\ddagger}$ & $0.35^{\ddagger}$ & $5.41^{* *}$ & NS & NS & NS & NS \\
\hline 1205 & $4.95^{\star \star}$ & $0.19^{\star}$ & $0.19^{\star}$ & $0.17^{\ddagger}$ & $0.35^{\ddagger}$ & $5.42^{\star \star}$ & NS & NS & NS & NS \\
\hline 1215 & $4.90^{\star \star}$ & $0.19^{\star}$ & $0.19^{*}$ & $0.17^{\ddagger}$ & $0.35^{\ddagger}$ & $5.41^{\star \star}$ & NS & NS & NS & NS \\
\hline 1225 & $4.84^{\star \star}$ & $0.19^{*}$ & $0.19^{\star}$ & $0.17^{\ddagger}$ & $0.36^{\ddagger}$ & $5.38^{* *}$ & NS & NS & NS & NS \\
\hline 1235 & $4.78^{\star \star}$ & $0.19^{\star}$ & $0.19^{*}$ & $0.18^{\ddagger}$ & $0.37^{\ddagger}$ & $5.37^{\star \star}$ & NS & NS & NS & NS \\
\hline 1245 & $4.73^{\star \star}$ & $0.19^{*}$ & $0.19^{*}$ & $0.18^{\ddagger}$ & $0.36^{\ddagger}$ & $5.35^{\star \star}$ & NS & NS & NS & NS \\
\hline 1255 & $4.68^{\star \star}$ & $0.18^{*}$ & $0.18^{*}$ & $0.18^{\ddagger}$ & $0.36^{\ddagger}$ & $5.34^{\star \star}$ & NS & NS & NS & NS \\
\hline 1265 & $4.65^{\star \star}$ & $0.19^{*}$ & $0.19^{*}$ & $0.18^{\ddagger}$ & $0.36^{\ddagger}$ & $5.32^{\star *}$ & NS & NS & NS & NS \\
\hline 1275 & $4.68^{\star \star}$ & $0.20^{*}$ & $0.20^{*}$ & $0.19^{*}$ & $0.38^{\ddagger}$ & $5.34^{\star \star}$ & NS & NS & NS & NS \\
\hline 1285 & $4.75^{\star \star}$ & $0.22^{*}$ & $0.22^{*}$ & $0.20^{*}$ & $0.40^{\ddagger}$ & $5.37^{\star \star}$ & NS & NS & NS & NS \\
\hline 1295 & $4.89^{* \star}$ & $0.24^{\star}$ & $0.42^{\star}$ & $0.21^{\star}$ & $0.42^{\ddagger}$ & $5.38^{\star *}$ & NS & NS & NS & NS \\
\hline 1305 & $5.00^{\star \star}$ & $0.27^{\star}$ & $0.45^{\star}$ & $0.22^{*}$ & $0.45^{\star}$ & $5.41^{\star \star}$ & NS & NS & NS & NS \\
\hline
\end{tabular}

† Year of release

$\S \mathrm{NS}=$ not statistically significant.

$\ddagger=\operatorname{Pr} \leq 0.15$.

${ }^{*}=\operatorname{Pr} \leq 0.05$.

${ }^{* *}=\operatorname{Pr} \leq 0.01$.

of the spectra were also highly correlated with maturity among the MGIII cultivars $(r=-0.48$ to -0.63$)$ and plant height among the MGIV cultivars ( $r=0.61$ to 0.73 ). Maturity increased as reflectance decreased in the VIS portion of the spectra among MGIII cultivars but not the MGIV cultivars. This could be due to MGIII cultivars having a greater separation of maturities among cultivars (15 d) compared to the MGIV cultivars (9 d). Plant height 

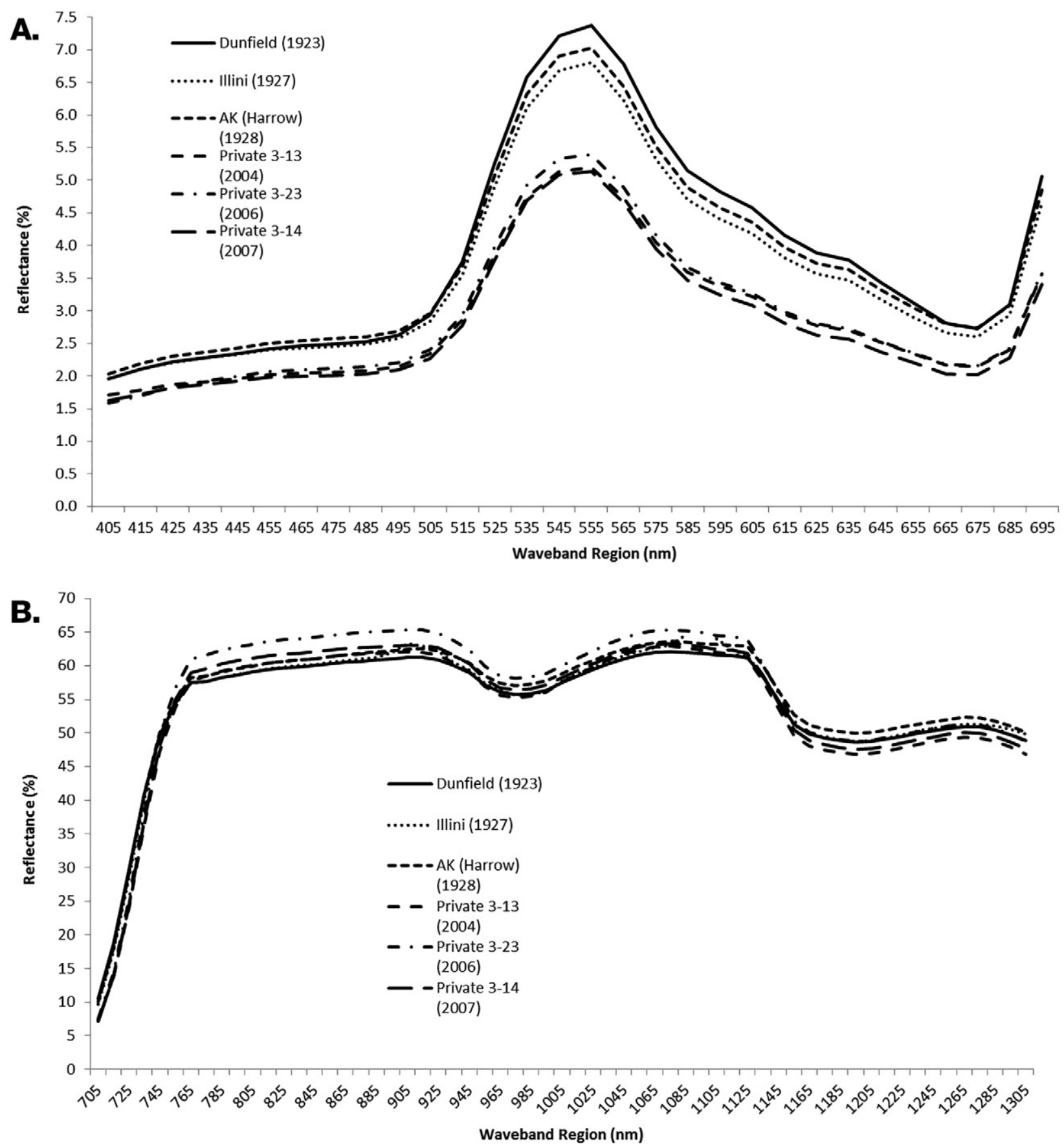

Figure 2. A. Mean spectral response curves for the 405-695 nm band regions of the three most recently-released and three earliestreleased maturity group III (MGIII) cultivars. B. Mean spectral response curves for the 705-1305 nm band regions of the three most recently-released and three earliest-released maturity group III (MGIII) cultivars. Wavebands are 10-nm intervals and reflectance is the percentage of a white reference panel.

increased as reflectance increased in the VIS portion of the spectra among MGIV cultivars.

Reflectance patterns in the NIR were not as consistent between maturity groups as the VIS patterns (Fig. 4 and 5). NIR reflectance among the MGIII cultivars was not related to YOR, except for the 1285-1305 nm region $(r=-0.46$ to -0.52$)$. Among the MGIV cultivars, NIR reflectance increased as YOR increased in the 765 to 1145 $\mathrm{nm}$ region. Maturity played a greater role among MGIV cultivars than MGIII cultivars in the NIR, with most of the NIR among MGIV cultivars being highly correlated with maturity whereas only a small area in the plateau region (735-895 nm) of the spectra being different among MGIII cultivars. Height and lodging were not significantly correlated to the NIR bands in both MGs, except for the $1105 \mathrm{~nm}$ waveband region among MGIII cultivars.

The reduced reflectance in the blue and red portions of the spectrum (405-495 and 605-695) with increases in YOR among the MGIII $(r=-0.61$ to -0.85$)$ and MGIV $(r=-0.53$ to -0.84$)$ cultivars could be due to changes in 

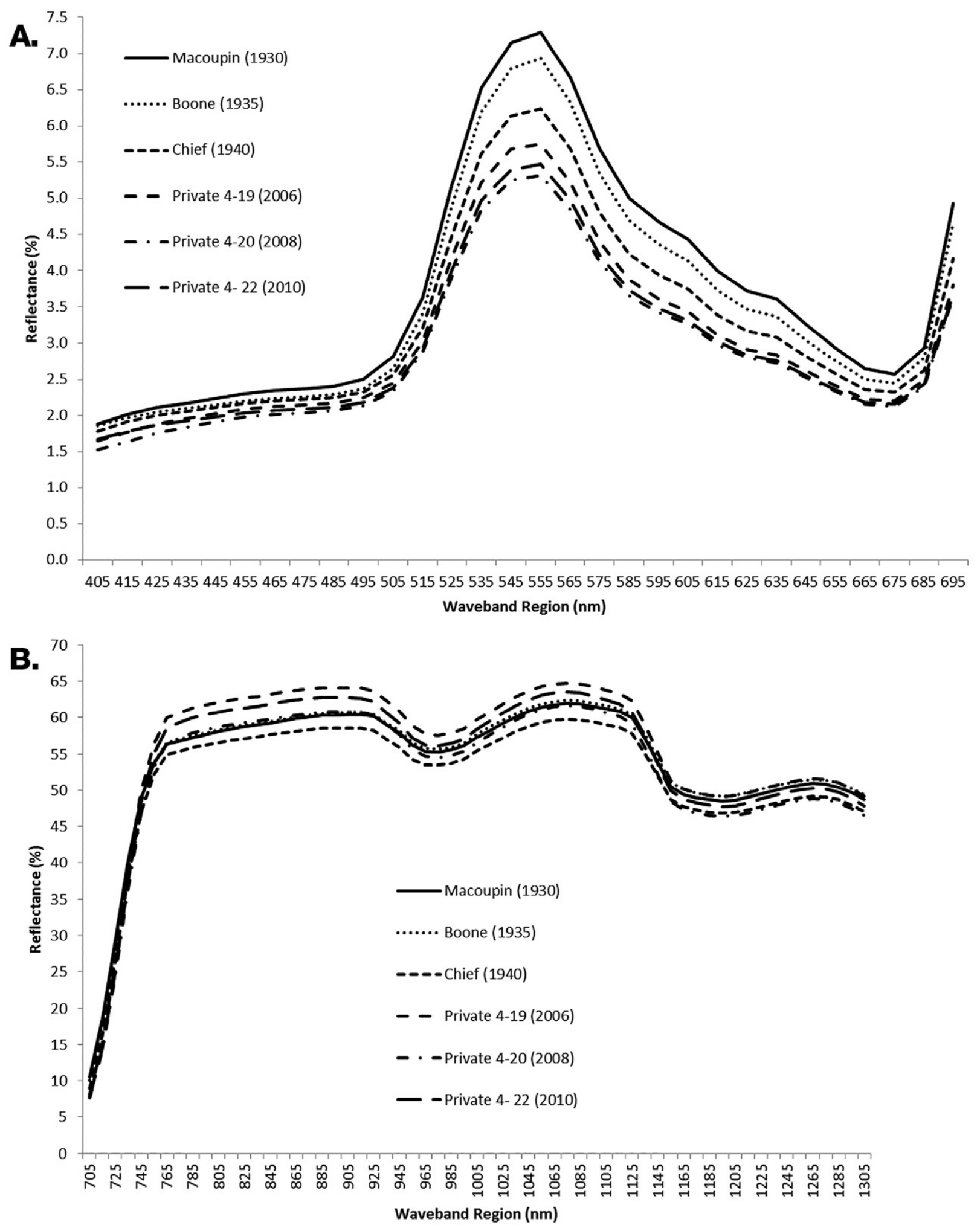

Figure 3. A. Mean spectral response curves for the 405-695 nm band regions of the three most recently-released and three earliestreleased maturity group IV (MGIV) cultivars. B. Mean spectral response curves for the 705-1305 nm band regions of the three most recently-released and three earliest-released maturity group IV (MGIV) cultivars. Wavebands are 10-nm intervals and reflectance is the percentage of a white reference panel.

the absorption peaks of chlorophyll $a$ and $b$ among cultivars (Chappelle et al., 1992). Chlorophyll $a$ and $b$ may be absorbing a greater amount of light in the more recent releases. In the red portions of the spectra, the positive relations with lodging among both MGs as well as height among MGIV cultivars, could be due to increased soil confounds from lodging and sparse canopy cover, causing increased reflection of red light. Soils highly reflect red light because 


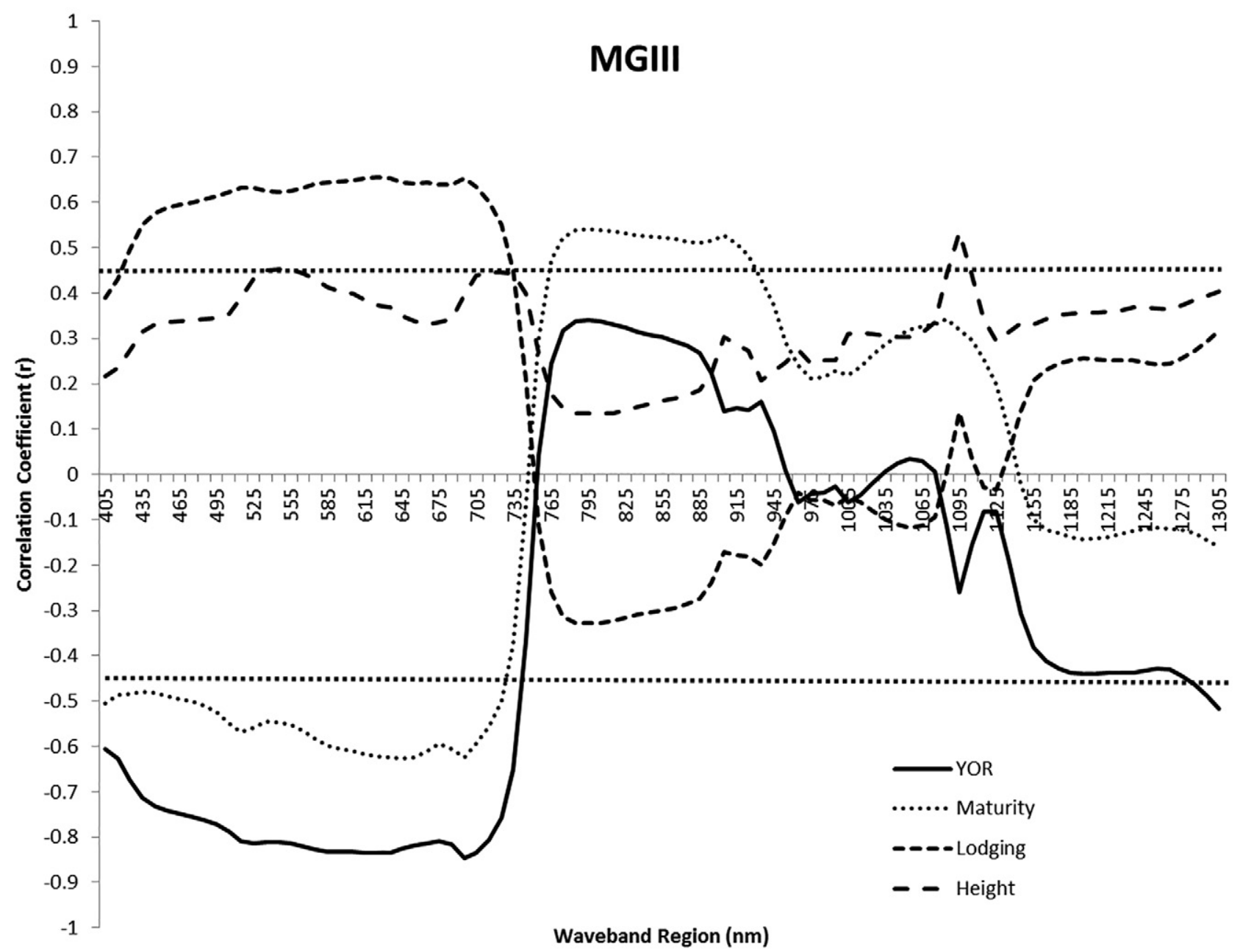

Figure 4. Correlation coefficient ( $r$ ) values between waveband regions (nm) and year of release (YOR), maturity, lodging, and height for maturity group III (MGIII) cultivar two-year mean reflectance values. Dotted lines represent significance at the 0.05 level.

of their mineral content. Negative correlations within the green portion of the spectra to YOR suggest more recently released cultivars are decreasing in green reflection percentages (Fig. 4 and 5). Higher reflection in the green has been correlated to increased yellow pigmentation, and therefore less healthy leaves/canopy due to stressors, increased disease and insect pressure, or cellular degradation (Sims and Gamon, 2002; Weber et al., 2012). The positive correlation among both MG cultivars between the green reflection percentages and lodging could suggest that increased lodging causes increased yellow pigmentation due to leaf structure breakdown and degradation. Also, among MGIV cultivars, increased height could correlate to increased yellow pigmentation, and therefore decreased canopy health and sparse canopy cover.

The strongest association between YOR and the spectral bands was observed in the red-edge region (705-735 $\mathrm{nm})$. Reduced reflection as YOR increased in this region resulted in correlations with YOR ranging between -0.76 and -0.88 , for MGIII and MGIV cultivars (Fig. 4 and 5). Significant relationships were also found between the rededge and maturity and lodging among MGIII cultivars and height and lodging but not maturity among MGIV cultivars. Filella and Peñuelas (1994) characterized the red-edge inflection point as the transition region between red and NIR, where a sharp increase in reflection values are observed. They found that the region correlated well with overall leaf/canopy health, in which the magnitude of the difference between the reflection values between the red and NIR is a function of chlorophyll content and health, plant nitrogen status, and plant water status. Plants with a steeper increase in reflection values between the two regions have high chlorophyll content, high leaf area, increased biomass, and overall healthier vegetation. Weber et al. (2012) found that the red-edge region contributed to yield estimation, accounting for a large portion of yield variation among corn cultivars. This study found negative relationships between YOR, maturity, and reflection values in this region. This could suggest greater plant/canopy health within more recently released cultivars as well as an increase in maturity, allowing the plants to stay greener, longer into the growing season. This may be due to stressors affecting earlier released cultivars more than more recently released MGIII cultivars. Red-edge 


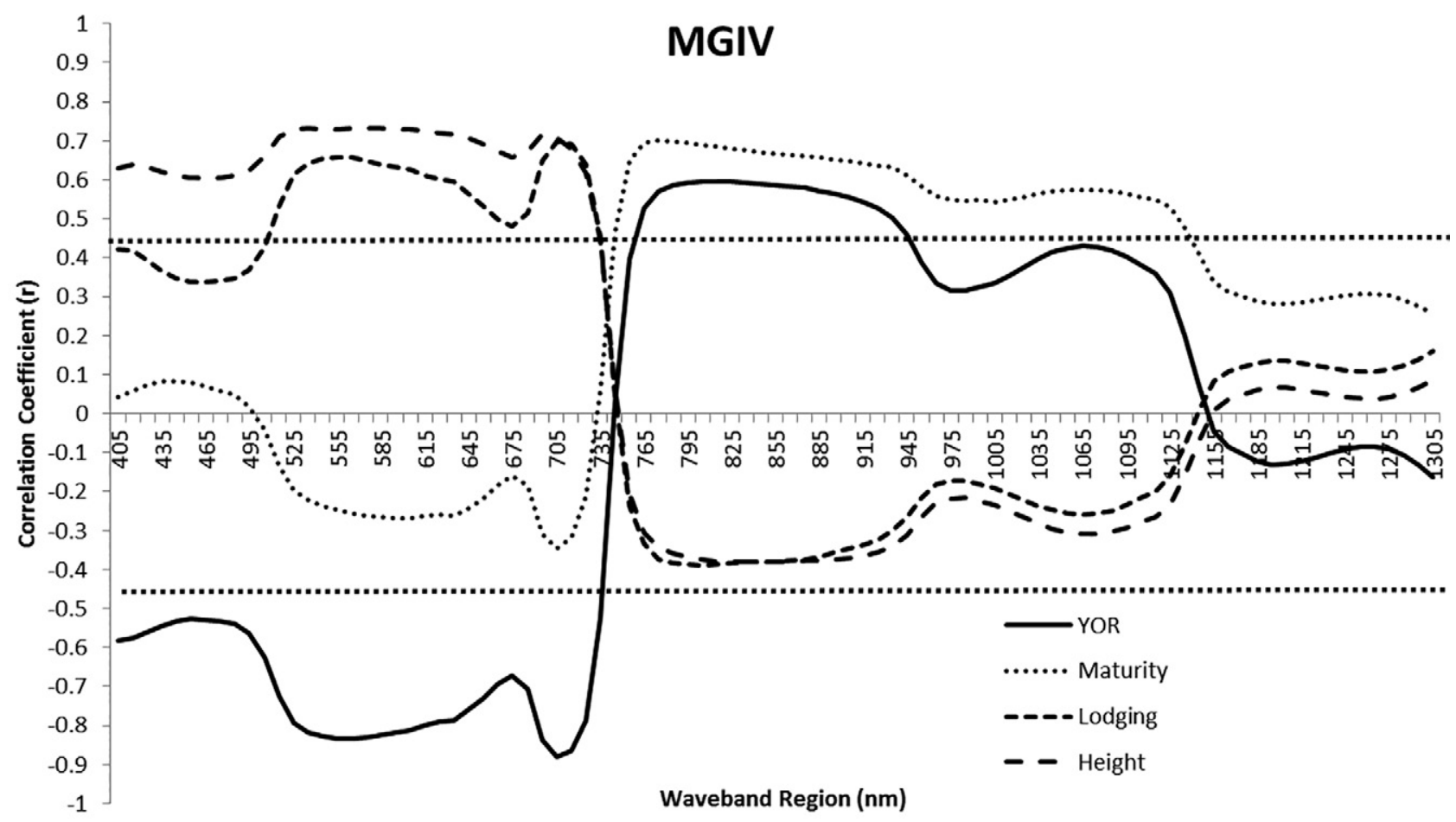

Figure 5. Correlation coefficient ( $r$ ) values between waveband regions ( $\mathrm{nm}$ ) and year of release (YOR), maturity, lodging, and height for group IV (MGIV) cultivar two-year mean reflectance values. Dotted lines represent significance at the 0.05 level.

reflectance among MGIV cultivars was not related to maturity; therefore, changes in reflectance patterns may be due to plant function alone. Increased lodging and height were associated with higher reflectance in the rededge region within MGIV cultivars, but only increased lodging showed higher reflectance among MGIII cultivars in this region.

Near-infrared measurements were not as well correlated with YOR as the VIS spectrum, but correlations were found between YOR and the NIR plateau $(735-895 \mathrm{~nm})$, as well as the small water absorption region (915-955 nm) among the MGIV cultivars (Fig. 4). However, no relationships were found between YOR and these waveband regions among MGIII cultivars (Fig. 5). Decreased spectral reflectance in the plateau region correlates with brown pigment (senescence), and correlations in both MGIII and MGIV cultivars suggests that maturity could have an effect in this region of the spectra. The maturity factor may be due to the extremely hot and dry conditions in 2011 and 2012 and an early frost in 2012, which prevented some MGIII and MGIV cultivars from reaching full maturity. Visual observations indicated that earlier-released MGIII and MGIV cultivars tended to have more foliar issues leading to early senescence and less green tissue due to disease than more recently released MGIII and MGIV cultivars. The plateau region (735-895 $\mathrm{nm}$ ) has been shown to be associated with total biomass production as well, which suggests that total biomass may have increased with YOR as well as increased with an increase in maturity or decreased with an increase in brown pigment accumulation (Weber et al., 2012). As in the plateau region, a portion of the small water absorption region (925-945 $\mathrm{nm}$ ) had a positive relationship between YOR among MGIV cultivars, and these band regions may suggest that total water content has increased as breeders have made selections based on other characteristics. Maturity also had relationships with waveband regions in this portion of the spectra, suggesting the later maturing, more recently-released cultivars could have higher water content, better overall biomass production, and healthier plant leaf structure later into the season than earlier-released cultivars.

This experiment found no association between reflectance in the 1100-1305 $\mathrm{nm}$ regions and YOR, maturity, height, or lodging among the MGIII or MGIV cultivars, except the 1285-1305 $\mathrm{nm}$ region among MGIII cultivars and YOR (Fig. 4 and 5). The 1100-1305 nm regions have been associated with plant water status, with high absorption and lower reflection of light by water leading to greater water content measurements within the plant leaf/canopy (Sims and Gamon, 2002). Sims and Gamon (2002) concluded that thick canopies (such as those seen in the more recently released cultivars) tend to confound water absorption regions, leading to increased reflection values. The greater reflectance values would be expected because less light penetrates thicker vegetation than thinner vegetation (Bull, 1991). Asrar et al. (1983) found that NIR and red reflectance could be used for leaf area index (LAI) calculations (which measures photosynthetically active tissue) and concluded that high reflectance in the NIR was associated with higher LAI values in wheat. However, LAI values are highly influenced by soil backgrounds, which could be an 
influence in the spectra obtained in this study and a reason for no correlations observed between wavebands and variables.

\section{Effect of Maturity, Height, and Lodging as Confounds with YOR}

These results have indicated that breeding advancement has impacted canopy spectral reflectance. However, differences observed among cultivars in reflectance associated with maturity, lodging, and plant height raise the possibility that the changes in canopy reflectance might be due to simple changes in these traits or the confound between all of these traits. To examine this possibility, PROC REG was used to characterize the relationship between the response variables: wavebands, and the predictor variables: YOR, maturity, plant height, and lodging in stepwise regression models. The results of two models are presented, the first with all variables having equal opportunity to be included in the model and predictor variables selected based on the strength of the predictor variable. The second tested whether YOR explained a significant amount of remaining variation when maturity, height, and lodging were forced into the model first. Results from these analyses are presented in Table 3. Each regression model was optimized with the four variables. This type of analysis does not separate confounding effect of the predictor variables but does test if YOR is a relevant variable when agronomic traits account for a portion of the variation among cultivars. The partial $R^{2}$ values are presented for YOR based on partial sums of squares analysis and the total model $R^{2}$ with contributions from YOR, maturity, lodging, and height.

Among MGIII cultivars, YOR was selected first and accounted for the largest amount of variation within optimized stepwise models in the visible and red edge portion of the spectra $(405-735 \mathrm{~nm})$ as well as the transition into the middle infrared region (1165-1305 nm). Among MGIV cultivars, YOR selection and importance was limited to portions of the green, red, and red edge portions of the spectra $(525-735 \mathrm{~nm})$. Among models where YOR was selected as a predictor variable, YOR accounted for 57 to 100\% (MGIII) and 61 to 100\% (MGIV) of the total variation explained by the model. When maturity, lodging, and plant height were forced into the regression models, YOR still accounted for a significant portion of the variation in the visible and red edge regions of the spectra with partial $R^{2}$ values ranging from 0.06 to 0.12 and from 0.06 to 0.23 among MGIII and MGIV cultivars, respectively. And in the 1085-1115 and 1175-1305 nm regions among MGIIIs, partial $R^{2}$ values ranged from 0.12 to 0.25 . These results indicated that spectral reflectance in these regions have changed with YOR, and therefore cultivar development through breeding advancement, with and without the impact of maturity, lodging, and height on variance explanation.

\section{CONCLUSIONS}

Differences in canopy spectral reflectance were found for the VIS (405-695 nm), red-edge (705-725 nm), and NIR (735-1305) waveband regions. The largest genotypic differences were observed in the red and red-edge portions of the spectra among both MGs. The NIR was more highly variable than the VIS and red edge, with fewer differences among cultivars in the NIR than VIS in both maturity groups.

Reflectance in the VIS and red-edge regions of the spectrum had the strongest negative correlations with YOR. The more-recently released cultivars tended to have lower reflectance values in the VIS and red-edge spectra portions and higher values in the NIR portion of the spectra than the earlier-released cultivars. These observations suggest a greater absorption of light incident on the leaves by the major pigments associated with photosynthesis, as well as an overall healthier leaf, canopy structure, and increased canopy cover/biomass production.

Reflectance patterns in certain wavelengths were also associated with maturity, lodging and plant height. However, when comparing variables within stepwise regression models, YOR continued to be an important predictor variable and explained a large portion of variation when maturity, lodging, and height were accounted for within the VIS and red-edge portions of the spectra for both MGs. However, YOR was not associated with spectral bands in the NIR beyond the $735 \mathrm{~nm}$ region until $1075 \mathrm{~nm}$ among MGIII cultivars, suggesting that differences in cultivars in this region were not associated with improvements in cultivar development.

Breeding has most affected spectral reflectance curves by reducing the VIS portion of the spectra and extending the red-edge, resulting in a shift to lower reflectance values further into the NIR and then a sharp inflection to the NIR. This suggests that the VIS and red edge regions of the spectra may be good candidates to be exploited for further cultivar development.

\section{Acknowledgments}

This is Contribution No. 13-375-J from the Kansas Agricultural Experiment Station, Manhattan. This research was supported in part by a grant from the Kansas Soybean Commission. The authors want to thank Dr. Brian Diers, University of Illinois, and Dr. James Specht, University of Nebraska, for providing seed of the soybean cultivars for this study.

\section{References}

Adams, M.L., W.D. Philpot, and W.A. Norvell. 1999. Yellowness index: An application of spectral 2nd derivatives to estimate chlorosis of leaves in stressed vegetation. Int. J. Remote Sens. 20:3663-3675. doi:10.1080/014311699211264

Asrar, G., M. Fuchs, E.T. Kanemasu, and J.L. Hatfield. 1983. Estimating absorbed photosynthetic radiation and leaf area index from spectral reflectance in wheat. Agron. J. 76:300-306. doi:10.2134/agronj1984.00021962007600020029x 
Babar, M., M.P. Reynolds, M. van Ginkel, A.R. Klatt, W.R. Raun, and M.L. Stone. 2006a. Spectral reflectance indices as a potential indirect selection criteria for wheat yield under irrigation. Crop Sci. 46(2):578-588. doi:10.2135/cropsci2005.0059

Babar, M.A., M.P. Reynolds, M. van Ginkel, A.R. Klatt, W.R. Raun, and M.L. Stone. 2006b. Spectral reflectance to estimate genetic variation for in-season biomass, leaf chlorophyll, and canopy temperature in wheat. Crop Sci. 46(3):1046-1057. doi:10.2135/cropsci2005.0211

Bull, C.R. 1991. Wavelength selection for near-infrared reflectance moisture meters. J. Agric. Eng. Res. 49:113-125. doi:10.1016/0021-8634(91)80032-A

Chang, J., D.E. Clay, K. Dalsted, S. Clay, and M. O’Neill. 2003. Corn (Zea Mays L.) yield prediction using multispectral and multidate reflectance. Agron. J. 95:1447-1453. doi:10.2134/ agronj2003.1447

Chappelle, E.W., M.S. Kim, and J.E. McMurtrey, III. 1992. Ratio analysis of reflectance spectra (RARS): An algorithm for the remote estimation of the concentrations of chlorophyll A, chlorophyll B, and carotenoids in soybean leaves. Remote Sens. Environ. 39:239-247. doi:10.1016/0034-4257(92)90089-3

Curran, P.J., J.L. Dungan, and D.L. Peterson. 2001. Estimating the foliar biochemical concentration of leaves with reflectance spectrometry: Testing the Kokaly and Clark methodologies. Remote Sens. Environ. 76:349-359. doi:10.1016/S00344257(01)00182-1

Curran, P.J., J.L. Dungan, and H.L. Gholz. 1990. Exploring the relationship between reflectance red edge and chlorophyll content in slash pine. Tree Physiol. 7:33-48. doi:10.1093/treephys/7.1-2-3-4.33

Datt, B. 1998. Remote sensing of chlorophyll $a$, chlorophyll $b$, chlorophyll $a+b$, and total carotenoid content in eucalyptus leaves. Remote Sens. Environ. 66:111-121. doi:10.1016/S00344257(98)00046-7

Datt, B. 1999. Remote sensing of water content in Eucalyptus leaves. Aust. J. Bot. 47:909-923. doi:10.1071/BT98042

Daughtry, C.S.T., C.L. Walthall, M.S. Kim, E. Brown de Colstoun, and J.E. McMurtrey, III. 2000. Estimating corn leaf chlorophyll content from leaf and canopy reflectance. Remote Sens. Environ. 74:229-239. doi:10.1016/S0034-4257(00)00113-9

Deering, D.W. 1978. Rangeland reflectance characteristics measured by aircraft and spacecraft sensors. Ph.D. diss., Texas A\&M Univ., College Station.

De Jong, S.M., E.J. Pebesma, and B. Lacaze. 2003. Above-ground biomass assessment of Mediterranean forests using airborne imaging spectrometry: The DAIS Peyne experiment. Int. J. Remote Sens. 24:1505-1520. doi:10.1080/01431160210145560

Dusek, D.A., R.D. Jackson, and J.T. Musick. 1985. Winter wheat vegetation indices calculated from combinations of seven spectral bands. Remote Sens. Environ. 18:255-267. doi:10.1016/00344257(85)90061-6

Fehr, W.R., and C.E. Caviness. 1977. Stages of soybean development. Spec. Rep. 80. Iowa Agric. Home Econ. Exp. Stn. Iowa State Univ., Ames.

Filella, I., L. Serrano, J. Serra, and J. Peñuelas. 1995. Evaluating wheat nitrogen status with canopy reflectance indices and discriminate analysis. Crop Sci. 35:1400-1405. doi:10.2135/crops ci1995.0011183X003500050023x

Filella, I., and J. Peñuelas. 1994. The red edge position and shape as indicators of plant chlorophyll content, biomass, and hydric status. Int. J. Remote Sens. 15(7):1459-1470. doi:10.1080/01431169408954177
Gamon, J.A., J. Peñuelas, and C.B. Field. 1992. A narrow-waveband spectral index that tracks diurnal changes in photosynthetic efficiency. Remote Sens. Environ. 41:35-44. doi:10.1016/0034-4257(92)90059-S

Gamon, J.A., and J.S. Surfus. 1999. Assessing leaf pigment content and activity with a reflectometer. New Phytol. 143:105-117. doi:10.1046/j.1469-8137.1999.00424.x

Gao, B.C. 1996. NDWI-a normalized difference water index for remote sensing of vegetation liquid water from space. Remote Sens. Environ. 58:257-266. doi:10.1016/S00344257(96)00067-3

Gitelson, A.A., and M.N. Merzlyak. 1996. Signature analysis of leaf reflectance spectra: Algorithm development for remote sensing of chlorophyll. J. Plant Physiol. 148:494-500. doi:10.1016/ S0176-1617(96)80284-7

Gitelson, A.A., and M.N. Merzlyak. 1994. Quantitative estimation of chlorophyll-a using reflectance spectra: Experiments with autumn chestnut and maple leaves. J. Photochem. Photobiol. 22:247-252. doi:10.1016/1011-1344(93)06963-4

Gutierrez, M., M.P. Reynolds, W.R. Raun, M.L. Stone, and A.R. Klatt. 2010. Spectral water indices for assessing yield in elite bread wheat genotypes under well-irrigated, water-stressed, and high-temperature conditions. Crop Sci. 50:197-214. doi:10.2135/cropsci2009.07.0381

Hansen, P.M., J.R. Jorgenson, and A. Thomsen. 2002. Predicting grain yield and protein content in winter wheat and spring barley using repeated canopy reflectance measurements and partial least squares regression. J. Agric. Sci. 139:307-318. doi:10.1017/ S0021859602002320

Kumar, R., and L. Silva. 1973. Light ray tracing through a leaf crosssection. Appl. Opt. 12:2950-2954. doi:10.1364/AO.12.002950

Lin, W.S., C.M. Yang, and B.J. Kuo. 2012. Classifying cultivars of rice (Oryza sativa L.) based on corrected canopy reflectance spectra data using the orthogonal projections of latent structures (O-PLS) method. Chemom. Intell. Lab. Syst. 115:25-36. doi:10.1016/j.chemolab.2012.04.005

Ma, B.L., L.M. Dwyer, C. Costa, E.R. Cober, and M.J. Morrison. 2001. Early prediction of soybean yield from canopy reflectance measurements. Agron. J. 93:1227-1234. doi:10.2134/ agronj2001.1227

Marti, J., J. Bort, G.A. Slafer, and J.L. Araus. 2007. Can wheat yield be assessed by early measurements of normalized difference vegetation index? Ann. Appl. Biol. 150:253-257. doi:10.1111/ j.1744-7348.2007.00126.x

Moran, J.A., A.K. Mitchell, G. Goodmanson, and K.A. Stockburger. 2000. Differentiation among effects of nitrogen fertilization treatments on conifer seedlings by foliar reflectance: A comparison of methods. Tree Physiol. 20:1113-1120. doi:10.1093/ treephys/20.16.1113

Morrison, M.J., H.D. Voldeng, and E.R. Cober. 1999. Physiological changes from 58 years of genetic improvement of shortseason soybean cultivars in Canada. Agron. J. 91:685-689. doi:10.2134/agronj1999.914685x

Peñuelas, J., F. Baret, and I. Filella. 1995. Semi-empirical indices to assess carotenoids/chlorophyll a ratio from leaf spectral reflectance. Photosynthetica 31:221-230.

Peñuelas, J., I. Filella, C. Biel, L. Serrano, and R. Save. 1993. The reflectance at the $950-970 \mathrm{~mm}$ region as an indicator of plant water status. Int. J. Remote Sens. 14:1887-1905. doi:10.1080/01431169308954010 
Peñuelas, J., R. Isla, I. Filella, and J.L. Araus. 1997. Visible and near infrared reflectance assessment of salinity effects on barley. Crop Sci. 37:198-202.

Prasad, B., B.F. Carver, M.L. Stone, M.A. Babar, W.R. Raun, and A.R. Klatt. 2007a. Genetic analysis of indirect selection for winter wheat grain yield using spectral reflectance indices. Crop Sci. 47:1416-1425. doi:10.2135/cropsci2006.08.0546

Prasad, B., B.F. Carver, M.L. Stone, M.A. Babar, W.R. Raun, and A.R. Klatt. 2007b. Potential use of spectral reflectance indices as a selection tool for grain yield in winter wheat under great plains conditions. Crop Sci. 47:1426-1440. doi:10.2135/cropsci2006.07.0492

Reynolds, M., Y. Manes, A. Izanloo, and P. Langridge. 2009. Phenotyping approaches for physiological breeding and gene discovery in wheat. Ann. Appl. Biol. 155(3):309-320. doi:10.1111/ j.1744-7348.2009.00351.x

Royo, C., N. Aparicio, D. Villegas, J. Casadesus, P. Monneveux, and J.L. Araus. 2003. Usefulness of spectral reflectance indices as durum wheat yield predictors under contrasting Mediterranean conditions. Int. J. Remote Sens. 24:4403-4419. doi:10.1080/0143116031000150059

Royo, C., D. Villegas, L.F. Garcia del Moral, S. Elhani, N. Aparicio, Y. Rharrabti, and J.L. Araus. 2002. Comparative performance of carbon isotope discrimination and canopy temperature depression as predictors of genotype differences in durum wheat yield in Spain. Aust. J. Agric. Res. 53:561-569. doi:10.1071/AR01016

SAS Institute. 2008. SAS 9.2. SAS Institute Inc., Cary, NC.

Serrano, L., I. Filella, and J. Peñuelas. 2000. Remote sensing of biomass and yield of winter wheat under different nitrogen supplies. Crop Sci. 40:723-731. doi:10.2135/cropsci2000.403723x
Shanahan, J.F., J.S. Schepers, D.D. Francis, G.E. Varvel, W.W. Wilhelm, J.M. Tringe, M.R. Schlemmer, and D.J. Major. 2001. Use of remote-sensing imagery to estimate corn grain yield. Agron. J. 93:583-589. doi:10.2134/agronj2001.933583x

Sims, D.A., and J.A. Gamon. 2002. Relationship between leaf pigment content and spectral reflectance across a wide range of species, leaf structures and development stages. Remote Sens. Environ. 81:337-354. doi:10.1016/S0034-4257(02)00010-X

Thomas, J.R., and H.W. Gausman. 1977. Leaf reflectance vs. leaf chlorophyll and carotenoid concentrations for eight crops. Agron. J. 69:799-802. doi:10.2134/agronj1977.000219620069 $00050017 \mathrm{x}$

Tucker, C.J. 1979. Red and photographic infrared linear combinations for monitoring vegetation. Remote Sens. Environ. 8:127150. doi:10.1016/0034-4257(79)90013-0

Viña, A., and A. Gitelson. 2011. Sensitivity to foliar anthocyanin content of vegetation indices using green reflectance. IEEE Geosci. Remote Sens. Lett. 8:464-468. doi:10.1109/ LGRS.2010.2086430

Weber, V.S., J.L. Araus, J.E. Cairns, C. Sanchez, A.E. Melchinger, and E. Orsini. 2012. Prediction of grain yield using reflectance spectra of canopy and leaves in maize plants grown under different water regimes. Field Crops Res. 128:82-90. doi:10.1016/j. fcr.2011.12.016

Wessman, C.A. 1990. Evaluation of canopy biochemistry. In: Remote Sensing of Biosphere Functioning, $1^{\text {st }}$ Edition, R. J. Hobbs and H. A. Mooney, Eds.,Ecological Studies 79, Springer Verlag, New York, p. 135-156.

Wiegand, C.L., A.J. Richardson, D.E. Escobar, and A.M. Gerbermann. 1991. Vegetation indexes for crop assessment. Remote Sens. Environ. 35:105-119. doi:10.1016/0034-4257(91)90004-P 